\title{
Development and validation of a synthetic 3D-printed simulator for training in neuroendoscopic ventricular lesion removal
}

\author{
${ }^{*}$ Maria Licci, MD, ${ }^{1,2}$ Florian M. Thieringer, MD, DDS, MHBA, ${ }^{3-5}$ Raphael Guzman, MD, ${ }^{1,2,5}$ and \\ Jehuda Soleman, MD1,2,5 \\ 1'Department of Neurosurgery, University Hospital of Basel; 'Division of Pediatric Neurosurgery, Children's University Hospital \\ of Basel; ${ }^{3}$ Department of Cranio-Maxillo-Facial Surgery, University Hospital Basel; ${ }^{43 D}$ Print Lab, University Hospital Basel; and \\ ${ }^{5}$ University of Basel, Switzerland
}

OBJECTIVE Neuroendoscopic surgery using an ultrasonic aspirator represents a valid tool with which to perform the safe resection of deep-seated ventricular lesions, but the handling of neuroendoscopic instruments is technically challenging, requiring extensive training to achieve a steep learning curve. Simulation-based methods are increasingly used to improve surgical skills, allowing neurosurgical trainees to practice in a risk-free, reproducible environment. The authors introduce a synthetic, patient-specific simulator that enables trainees to develop skills for endoscopic ventricular tumor removal, and they evaluate the model's validity as a training instrument with regard to realism, mechanical proprieties, procedural content, and handling.

METHODS The authors developed a synthetic simulator based on a patient-specific CT data set. The anatomical features were segmented, and several realistic 1:1 skull models with all relevant ventricular structures were fabricated by a 3D printer. Vascular structures and the choroid plexus were included. A tumor model, composed of polyvinyl alcohol, mimicking a soft-consistency lesion, was secured in different spots of the frontal horn and within the third ventricle. Neurosurgical trainees participating in a neuroendoscopic workshop qualitatively assessed, by means of a feedback survey, the properties of the simulator as a training model that teaches neuroendoscopic ultrasonic ventricular tumor surgery; the trainees rated 10 items according to a 5-point Likert scale.

RESULTS Participants appreciated the model as a valid hands-on training tool for neuroendoscopic ultrasonic aspirator tumor removal, highly rating the procedural content. Furthermore, they mostly agreed on its comparably realistic anatomical and mechanical properties. By the model's first application, the authors were able to recognize possible improvement measures, such as the development of different tumor model textures and the possibility, for the user, of creating a realistic surgical skull approach and neuroendoscopic trajectory.

CONCLUSIONS A low-cost, patient-specific, reusable 3D-printed simulator for the training of neuroendoscopic ultrasonic aspirator tumor removal was successfully developed. The simulator is a useful tool for teaching neuroendoscopic techniques and provides support in the development of the required surgical skills.

https://thejns.org/doi/abs/10.3171/2019.12.FOCUS19841

KEYWORDS neuroendoscopy; ultrasonic surgery; ventricular tumor; simulation-based training; 3D printing; additive manufacturing

$\mathrm{N}$ EUROSURGICAL education programs to acquire surgical skill require extensive training and hands-on experience to develop high levels of competence and achieve a steep learning curve. Intensive surgical exposure, in turn, requires a large operative case volume. In an era of decreasing operative exposure during residen$\mathrm{cy}-\mathrm{a}$ trend that has been influenced, among other factors, by resident work-hour restrictions, medicolegal and ethical concerns of hands-on treatment of actual patients, and reduced time availability for surgical instruction in favor of cost-effective operating room practices-interest in simulation-based medical education has been growing. ${ }^{18}$ Furthermore, the increased subspecialization of surgeons, changes in disease management, and technological ad-

ABBREVIATIONS ETV = endoscopic third ventriculostomy; VR = virtual reality.

SUBMITTED October 31, 2019. ACCEPTED December 20, 2019.

INCLUDE WHEN CITING DOI: 10.3171/2019.12.FOCUS19841.

${ }^{*}$ R.G. and J.S. share senior authorship of this work. 
vances, paired with an increased demand for patient safety in the delivery of care, have led surgical educators to seek out alternative training methods over the years, thus incorporating surgical simulation into the surgical skill training programs and courses for postgraduate surgical education. . $^{3,-9,18}$ Competence-based education plays an essential role in the training of neuroendoscopic procedures, as the handling of endoscopic instruments entails a steep learning curve, even for surgeons who are accustomed to microsurgery. In neuroendoscopy, as a minimally invasive surgical technique, the surgeon faces the danger of becoming disoriented with the endoscope and of possibly losing sight of anatomical landmarks. Furthermore, it presupposes the need for dual-handed maneuverability, additional skills for eye-hand coordination, and the adaptation of 2D visualization. ${ }^{5,16}$ Different models are used in surgical training, namely cadaveric and animal models, synthetic simulators, and virtual reality (VR) settings. Classically, cadaveric dissection has been the state of the art for anatomical learning. The absence of ventriculomegaly in most cadaveric specimens, however, limits its application for neuroendoscopy, making the cadavers more suitable for microsurgical training as opposed to endoscopic procedures. ${ }^{15}$ In addition, cadaveric dissection is limited by ethical and financial concerns. Animal models, while well accepted for use in other surgical fields, have ethical concerns and high maintenance costs and do not compare well anatomically with human brains in neuroendoscopic training. All the aspects noted have motivated the development of simulation-based educational tools. Simulators have been shown to further improve the level of specific clinical skill acquisition goals compared to traditional clinical medical education. Hence, simulation has gained significance in exposing trainees to various procedures before they apply them in the actual patient population..$^{21,23}$ A new trend, correlating with rapid technical advances, is shown by the development of VR systems, in addition to $3 \mathrm{D}$-printed anatomical simulators. ${ }^{8}$ Overall, simulationbased training tools, including realistic 3D-printed models and VR settings, are free from concerns over patient safety, and they provide the advantage of a no-risk training environment for repetitive practice. Furthermore, other advantages are their portability and the reusability of their components, as well as their cost-effectiveness with regard to 3D-printed models. However, they lack a realistic surface and tissue properties for anatomical dissection and might not be able to simulate complex procedures. ${ }^{7,25}$

To address the need for training surgeons in how to perform neuroendoscopic ventricular lesion removal in particular, we developed and validated a patient-specific, low-cost, 3D-printed simulator that can be used repeatedly to increase familiarity with endoscope handling and to train coordinative skills.

\section{Methods}

\section{Skull and Ventricle Simulator Design and Construction}

To fabricate the 3D-printed models for this study in the University Hospital Basel's 3D Print Lab ${ }^{24}$ a representative, anonymized CT data set of a patient with enlarged CSF spaces was first downloaded from the picture ar- chiving and communication system (PACS) and further processed with the medical segmentation software Materialise Mimics (Mimics Innovation Suite v20; Materialise). The DICOM CT data set consisted of native crosssectional slices of bone and soft-tissue windows to display the relevant anatomical features. Further processing and segmentation of several anatomical structures according to tissue density (Hounsfield units) was worked out. In particular, the strongly contrasted ventricular system could be displayed separately. The virtual cranial vault was designed with the help of the modeling software Materialise 3-Matics to be removable and equipped with realistic, neurosurgical burr holes for endoscopic access (Fig. 1). The osseous skull was printed completely (2 parts) with a consumer Replicator+ 3D printer (MakerBot Industries) from polylactic acid (PLA; light gray), and the corresponding ventricle spaces were divided into 2 parts with a wall thickness of $3 \mathrm{~mm}$ in transparent PLA material. After printing a total of 5 skull models, the support structures were manually removed, and the two halves of the ventricular system were glued together (UHU Kunststoffkleber hart; UHU GmbH \& Co. KG). These were inserted into the skull model, and the cavity between the ventricular system and the bony skull was filled with 2-component silicone for stabilization. This made it possible to ensure that the ventricular cavity was watertight (Fig. 2).

\section{Tumor Model Design and Construction}

We collaborated with Söring GmbH, generating a tumor model manufactured of polyvinyl alcohol, whose mixture properties are alterable during the manufacturing process so that various densities can be reached. For educational purposes, we chose a soft texture, facilitating fragmentation with the endoscopic ultrasonic instrument. The artificial lesions were implanted and secured to the walls of the frontal horn and within the third ventricle using instant glue, in order to meet the need for tissue attachment and guarantee adherence in a fluid-filled cavity (Fig. 3).

\section{Cost Analysis for the Fabrication of the 3D-Printed Model}

In order to analyze the manufacturing costs of 3Dprinted neurosurgical training models, it is necessary to consider the cost of software, hardware, and labor. Energy consumption and other infrastructure costs can be set aside. The cost framework for software licenses amounts to several thousand US dollars per year, but it must be mentioned that the 3D training models could also be produced with several open-source software applications. Here, the workflow would be more cumbersome and correspondingly more time-consuming. For the time of segmentation, modeling in several iterations, and preparation of the prototype model for printing, approximately 10-15 hours of working time is estimated; however, the renewed production of the models would now take considerably less time (approximately 4-5 hours) due to the established workflow. For printing, a Makerbot Replicator+ 3D FFF printer was used, which currently costs approximately $\$ 2500$ in US dollars. In total, materials were processed for $\$ 94$ per 3D-printed training model. The working time 

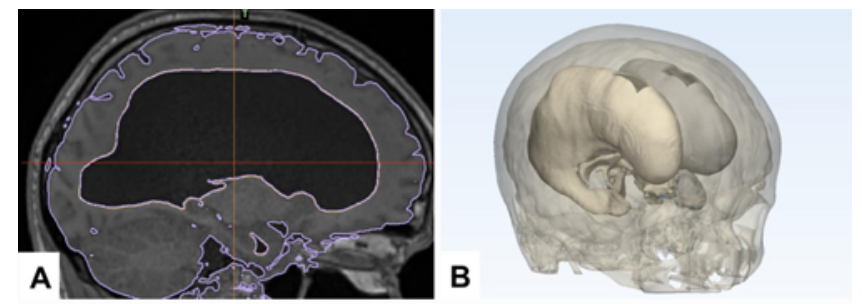

C

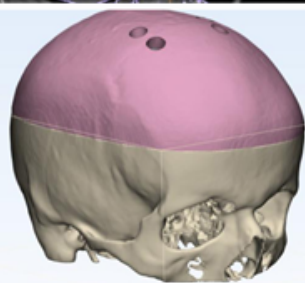

D

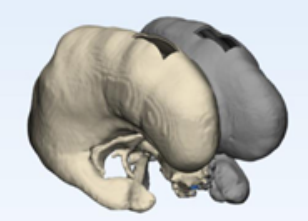

FIG. 1. A: Sagittal section of multimodal CT data with contour of segmented image data, showing a hydrocephalic lateral ventricle. B: 3D view of the osseous skull, showing a projection of the CSF ventricle system with cranial windows for endoscopy. C: Two-part osseous skull model ready for 3D printing. D: Two-part ventricle model prepared for $3 \mathrm{D}$ printing.

for the production of a model from a 3D data set, including postprocessing, is approximately $1-2$ hours per model. The models and digital data sets are reusable, and thus the production costs and time required can be subsequently significantly reduced during production.

\section{Hands-On Evaluation of the Simulator and Data Collection}

The neuroendoscopic ultrasonic tumor removal simulator was first designed for and introduced to residents taking part in a hands-on training program at our annual Pediatric Neurosurgery Symposium, being organized at the University Children's Hospital in Basel, Switzerland, in 2019, as part of a skill education course, including, additionally, the performance of endoscopic third ventriculostomy (ETV) on cadaveric specimens and the application of preoperative planning tools using virtual augmented reality. Prior to using the 3D-printed simulator for training in neuroendoscopic ventricular lesion removal during a training course for neurosurgical residents, the model was tested by 4 board-certified neurosurgeons of different international institutions, at either the senior consultant or chairman level. This was helpful for improving the setup of the training course. The handling and the anatomical realism were appreciated by all 4 neurosurgeons. However, we did not seek their opinions with the structured survey, though we still gained important information about weaknesses of the model from their open comments. The ventricular model, provided with a $1 \times 2-\mathrm{cm}$ entry point in the frontal horn, placed in the projection of the foramen of Monro, was filled with $\mathrm{NaCl}$ solution and inserted in the skull model, which had burr holes made at Kocher's point bilaterally, simulating a typical trajectory for the frontal placement of a ventricular drain. Participants were asked to perform endoscopic ultrasonic resection of the ventricular lesions using the Endoscopic Neurosurgical Pen (ENP) (Söring GmbH), an endoscopic ultrasound aspirator whose long sonotrode is guided through the working channel of a GAAB endoscope (28096 AGA, trocar: 28162 BS; KARL STORZ), complemented with an ultrasonic generator (SONOCA 300; Söring GmbH). This technique allows for fragmentation and simultaneous aspiration of a lesion, facilitating minimally invasive surgery for deep-seated lesions (Fig. 4; Video 1).

VIDEO 1. Intraventricular view of progressive ultrasonic tumor aspiration in the right frontal horn using the Söring Endoscopic
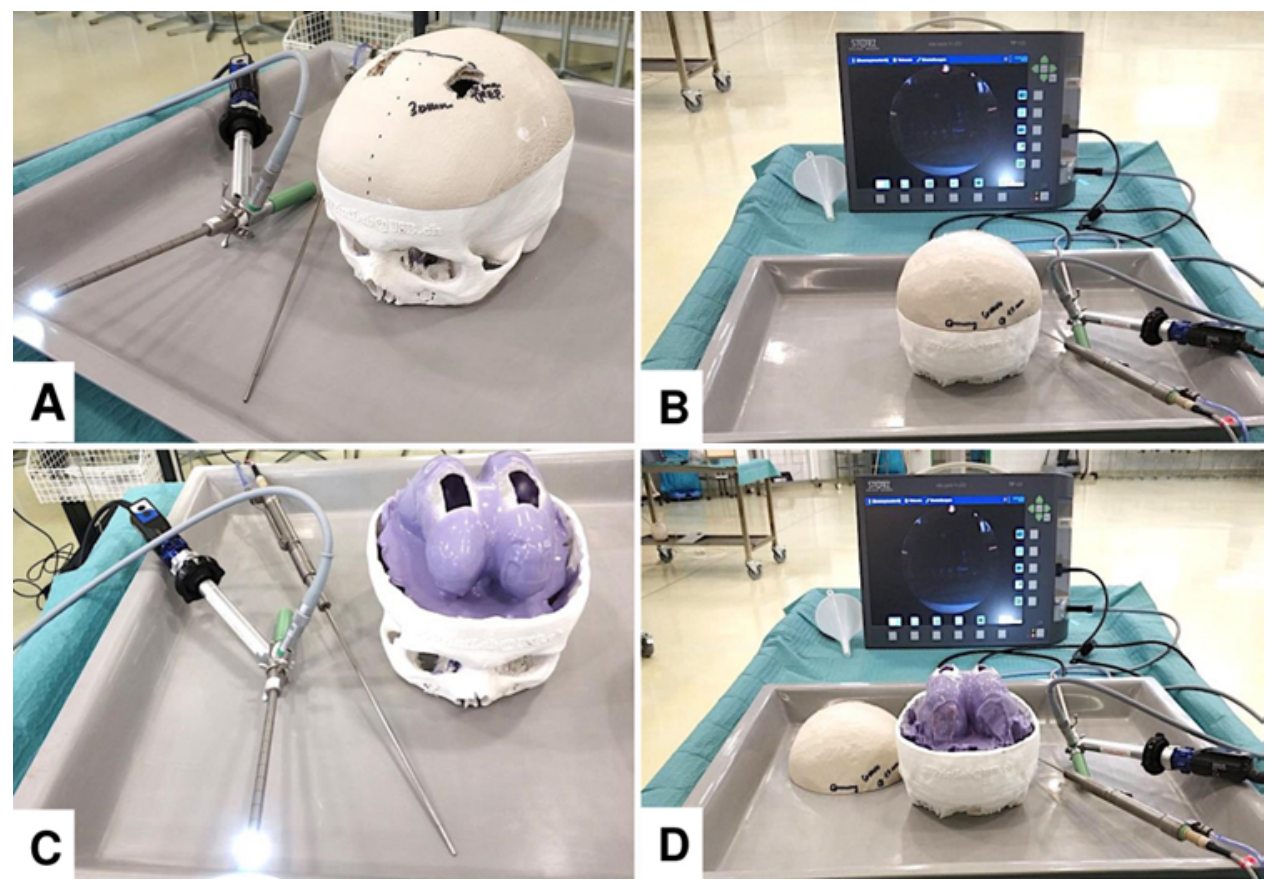

FIG. 2. Simulator training setup with skull model (A), ventricular model (C), GAAB Endoscope, Söring Endoscopic Neurosurgical Pen, xenon light source, and monitor with digital video recording system (B and $\mathbf{D})$. 


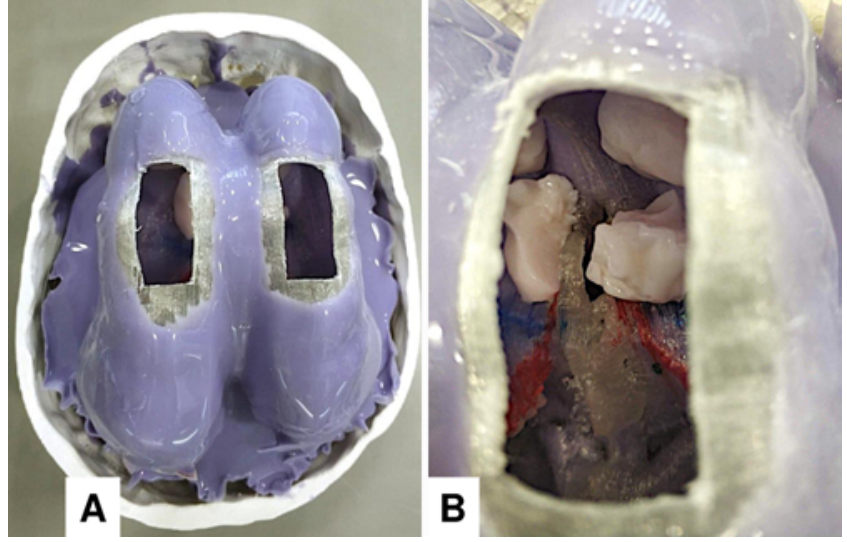

FIG. 3. A: Ventricular model overview with right frontal horn shown. B: Detailed view of the ventricular content through a frontal horn opening, showing tumor models in the lateral ventricle, thalamostriate vein, anterior septal vein, and plexus choroideus.

Neurosurgical Pen. Copyright Jehuda Soleman. Published with permission. Click here to view.

Upon completion of the course, an online survey comprising 10 questions pertaining to handling and teaching effectiveness of the neuroendoscopic simulator was sent to the participants. For data collection, residents of Swiss and German neurosurgery training programs were selected from among the participants. They were asked to rate their level of agreement with each item on the questionnaire by means of a 5-point Likert scale, with the following scores: 1 , strongly disagree; 2 , disagree; 3 , neutral; 4, agree; and 5, strongly agree. Incomplete questionnaires were excluded, resulting in the analysis of 10 complete evaluation forms.

\section{Results}

The results of the feedback survey are summarized in Table 1. All those surveyed agreed or strongly agreed (Likert scores 4 and 5) on the model's realistic anatomical properties of the skull and ventricular system. Comparably, Likert scores of 4 and 5 were given regarding the technical proprieties of the model. In fact, participants rated the camera view as comparable to a real surgical setting (Likert scores 4 and 5). Lastly, the participants agreed or strongly agreed that the content validity of the simulator was a useful tool to increase surgical competency for neuroendoscopic procedures in that it helped to develop coordinative skills and represented a valuable practical exercise tool for ventricular tumor removal. Weaknesses of the model were indicated by neutral responses on the representativeness of the model as a realistic tumor removal exercise tool. However, most of those surveyed (70\%-80\%) stated that the handling of the neuroendoscopic ultrasonic simulator during tumor removal was comparably realistic with regard to mechanical proprieties, namely tactile feedback and response to manipulation.

\section{Discussion}

Changes in surgical education, driven by work-hour restrictions, ethical and medicolegal concerns for patient safety, and financial demands of increased operating room efficiency, have led to limited teaching time for surger-

TABLE 1. Summary of the questionnaire for simulator validity assessment

\begin{tabular}{|c|c|c|c|c|c|}
\hline Questionnaire Item & $\begin{array}{c}\text { Strongly } \\
\text { Disagree (1) }\end{array}$ & Disagree (2) & Neutral (3) & Agree (4) & $\begin{array}{l}\text { Strongly } \\
\text { Agree (5) }\end{array}$ \\
\hline $\begin{array}{l}\text { 1) The 3D-printed skull model had appropriate surface anatomy w/ a realistic } \\
\text { entry point \& suitable trajectory for the endoscope }\end{array}$ & $0(0 \%)$ & $0(0 \%)$ & $0(0 \%)$ & $3(30 \%)$ & $7(70 \%)$ \\
\hline $\begin{array}{l}\text { 2) The ventricular system was realistic \& appropriately detailed for neuroendo- } \\
\text { scopic orientation }\end{array}$ & $0(0 \%)$ & $0(0 \%)$ & $0(0 \%)$ & $9(90 \%)$ & $1(10 \%)$ \\
\hline $\begin{array}{l}\text { 3) The ventricular tumor was realistic \& had appropriate details required to } \\
\text { perform the neuroendoscopic resection (ventricular wall adherence, location, } \\
\text { size, texture) }\end{array}$ & $0(0 \%)$ & $0(0 \%)$ & $2(20 \%)$ & $7(70 \%)$ & $1(10 \%)$ \\
\hline $\begin{array}{l}\text { 4) Performing the neuroendoscopic ultrasonic intraventricular tumor removal } \\
\text { w/ the simulator was realistic (tactile feedback, response to manipulation, } \\
\text { extirpation) }\end{array}$ & $0(0 \%)$ & $0(0 \%)$ & $2(20 \%)$ & $7(70 \%)$ & $1(10 \%)$ \\
\hline 5) The camera view is comparable to that seen in a real surgical setting & $0(0 \%)$ & $0(0 \%)$ & $0(0 \%)$ & $8(80 \%)$ & $2(20 \%)$ \\
\hline $\begin{array}{l}\text { 6) Skills to handle the endoscope \& the ultrasonic instrument were representa- } \\
\text { tive of those required to perform the real procedure }\end{array}$ & $0(0 \%)$ & $0(0 \%)$ & $3(30 \%)$ & $7(70 \%)$ & $0(0 \%)$ \\
\hline $\begin{array}{l}\text { 7) The simulator helps to develop the coordinative skills needed for neuroen- } \\
\text { doscopy }\end{array}$ & $0(0 \%)$ & $0(0 \%)$ & $0(0 \%)$ & $1(10 \%)$ & $9(90 \%)$ \\
\hline $\begin{array}{l}\text { 8) The neuroendoscopic ultrasonic surgery tumor removal task is a valuable } \\
\text { training exercise }\end{array}$ & $0(0 \%)$ & $0(0 \%)$ & $0(0 \%)$ & $2(20 \%)$ & $8(80 \%)$ \\
\hline $\begin{array}{l}\text { 9) Use of this model helps to increase competency when applied to train endo- } \\
\text { scopic ventricular tumor removal }\end{array}$ & $0(0 \%)$ & $0(0 \%)$ & $0(0 \%)$ & $3(30 \%)$ & $7(70 \%)$ \\
\hline $\begin{array}{l}\text { 10) I feel more confident using neuroendoscopic instruments for tumor removal } \\
\text { after training } w / \text { this model }\end{array}$ & $0(0 \%)$ & $0(0 \%)$ & $2(0 \%)$ & $5(50 \%)$ & $3(30 \%)$ \\
\hline
\end{tabular}




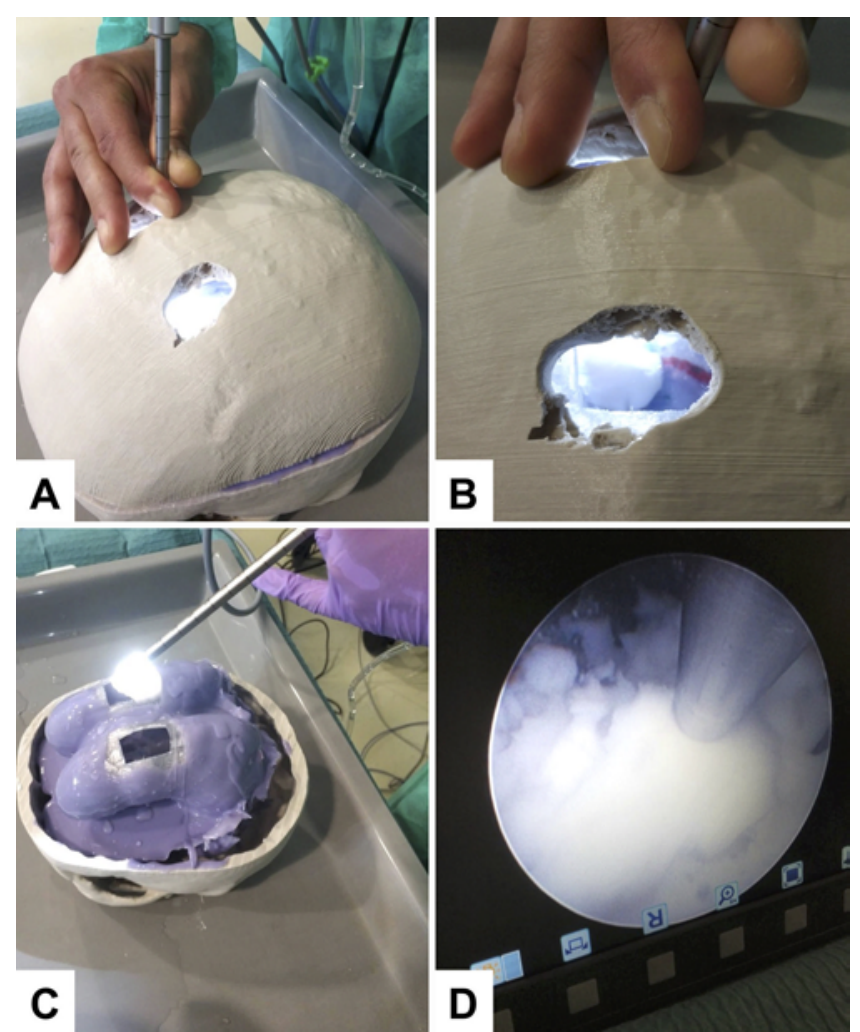

FIG. 4. Simulator training hands-on overview (A) and detailed view of instrument handling through the skull model (C). Detail of the ventricular model to illustrate the trajectory (B). View during endoscopic ultrasonic fragmentation of the tumor model (D).

ies in operating theaters. ${ }^{5-10,12,18}$ In turn, surgical training requires a long learning curve, and thus a comparison of these realities reveals the conflict between the need for education on the one hand and efficiency combined with avoidance of complications on the other hand. Incorporation of neurosurgical training models that simulate live surgery in training programs and workshops has been growing over time, with cadaveric and animal model dissection courses representing established methods. In 2002, Aboud et al. presented a cadaveric model in which vessels were filled with colored fluid that mimicked not only normal human anatomy but also dynamic vascular filling. ${ }^{1}$ Cadaveric models, however, lack ventriculomegaly in most specimens and might therefore be unsuitable for the training of neuroendoscopic procedures. Nevertheless, there are injectable cadaveric intraventricular tumor models for the training of navigation-guided endoscopic resection, challenging the trainee with pathologically distorted anatomy and allowing skill development for ventricular tumor resection, despite the absence of hydrocephalus. ${ }^{4,17}$ The use of animal models, by contrast, is weakened by the poor anatomical similarity to human anatomy. However, such models are highly valuable in that they allow the training of endoscopic neurosurgical port techniques and instrument handling, shortening the length of the steep learning curve and supporting the development of microsurgical dissection techniques, as described by Fernandez-Miranda et al. ${ }^{14}$ Moreover, Jaimovich et al. devel- oped an animal neuroendoscopy model that allows the use of the intraperitoneal cavity in Wistar rats, generating a hydroperitoneum, which allows navigation of the cavity content and the training of neuroendoscopic procedures, including the resection of a bleeding tumor (liver). ${ }^{19} \mathrm{With}$ the advancement of technology and the progressive call for simulation models, allowing repeated use in a riskfree environment, synthetic simulators and VR techniques have increasingly emerged in recent years. The original aim of VR models was to conceptualize the ventricular anatomy and surrounding neuronal structures to provide for an accurate 3D reconstruction for purposes of anatomical education. ${ }^{2,20}$ Furthermore, VR models are gaining acceptance as platforms for technical skill training, with systems having also been developed for ventriculostomy and endoscopic surgery. For this purpose, NeuroTouch is an integrated platform, simulating an operating room microscopic view and the $2 \mathrm{D}$ indirect endoscopic view. ${ }^{10,13}$ Whereas VR models might show relative benefits compared with synthetic simulators, most VR systems have the disadvantage of insufficiently conveying the complexity of the intervention, are expensive, and, above all, do not offer the tactile feedback of synthetic simulators. Hence, concerning realistic anatomical representation, synthetic simulators - the category under which our model fallsare stronger when it comes to the training of instrument handling and procedural content. ${ }^{8}$ However, most existing synthetic simulators were designed primarily for the training of ventriculostomy. $7,15,16,22,25,26$

One further model was generated for endoscopic colloid cyst resection with a replaceable, cystic lesion of variable viscosity, protruding through either of the foramina of Monro. ${ }^{6}$ Moreover, the Brazilian group of Zymberg et al. developed the reusable S.I.M.O.N.T. (Global Technologies), a real simulator that can be used for neuroendoscopic, rhinological, and endonasal skull base surgery. ${ }^{26}$ To be considered relevant for the purpose of comparison with our model and its evolution, physical simulators need to include or focus on neuroendoscopic tumor specimens, like the S.I.M.O.N.T. and the A.S.P.E.N., which was developed for the pediatric population. . $^{11,12,15,26}$ Advanced model simulators were provided with multiple ventricular lesions and were assessed by novices and experienced neurosurgeons, allowing also for CT and MRI analysis and association with image-guided navigation. Furthermore, the simulator includes bleeding-effect simulation, tumoral lesions with different textures, and a choroid plexus that can be cauterized.

To our knowledge, the presented model is the first synthetic simulator used for the training of neuroendoscopic ultrasonic tumor removal. Its core strength lies in its low cost and the rapid production of its components, as well as its reusability, except for the tumor sample, which needs to be replaced after each completed procedure. The patient specificity of the 3D-printing technology could be of use for surgery planning in selected cases.

During the hands-on training session and the questionnaire evaluation, some limitations of the model emerged and need to be addressed for further development. First, it is worth mentioning that it is not possible to plan a frontal approach for the insertion of a ventricular drain (e.g., 
definition of the skin incision, burr hole placement, and trajectory definition passing through brain parenchyma), albeit its elementary importance in training young neurosurgeons on a resident's level on the one hand and the resulting unrealistic wide range of motion (of the simulator's approach) for endoscope manipulation on the other hand. Second, the focus of our synthetic model was to give trainees neuroendoscopic skills for ultrasonic ventricular tumor removal, and although all surveyed participants agreed on its face validity, there was a preponderance of agreement, rather than strong agreement, regarding the model's mechanical proprieties, such that technical refinements were encouraged. For example, the simulator should be provided with a continuous irrigation tool to be comparable with the real-life endoscopic ventricular surgery setting, and a heterogeneous texture of the artificial tumor could reliably mimic real pathologies, such as cystic and solid lesions. Furthermore, the rigid ventricular wall consistency distorted realistic wall adherence and invasion of the tumor model, calling for more elastic and permeable material proprieties that would allow for liquid fluctuation and ventricular tension comparable to those seen in a real anatomical setting. Moreover, correction of the aforementioned technical limitations would help in allowing the model's extended use as a tool with which to practice ETV. Currently, our group is at work on the design of a ventricular model fully made of soft silicone, with appropriately colored anatomical structures, a ventricular wall consistency that adequately mimics ependymal lining tension and pulsation, and a thin floor able to simulate the conditions required for realistic endoscope manipulation, performance of ETV, and cyst resection. In terms of cost-effectiveness, we do not collect detailed data on the acquisition and maintenance costs of both cadaveric and animal models at our institution, but the low material costs of the simulator model are likely to be strongly competitive. Moreover, since both models and digital data sets are reusable, there is a significant potential for further cost and time reductions during manufacturing.

\section{Conclusions}

In an era of rapid technical advancement and growing interest in simulation-based surgical training, we developed a realistic, patient-specific, 3D-printed, portable, and reusable simulator for skill development in neuroendoscopic ultrasonic tumor removal. These features, along with the low material cost, make it suitable for neurosurgical training purposes. Beyond the technical characteristics of the simulator, the survey respondents' rating of the model's hands-on application during a neuroendoscopic workshop indicated satisfactory results for both procedural and content components. The strength of the simulator is its integrated tumor tissue, which is suitable for training on the use of ultrasonic aspiration. Nevertheless, further developments are required for improvement, including the adaption of material characteristics and expansion of application possibilities to a broader spectrum of neuroendoscopic procedures.

\section{Acknowledgments}

We particularly thank Ms. Carina Tooren, Product Manager,
Ultrasonic Surgery, Söring GmbH, for technical support and development of the tumor model.

\section{References}

1. Aboud E, Al-Mefty O, Yaşargil MG: New laboratory model for neurosurgical training that simulates live surgery. J Neurosurg 97:1367-1372, 2002

2. Adams CM, Wilson TD: Virtual cerebral ventricular system: an MR-based three-dimensional computer model. Anat Sci Educ 4:340-347, 2011

3. Agrawal A, Kato Y, Sano H, Kanno T: The incorporation of neuroendoscopy in neurosurgical training programs. World Neurosurg 79 (2 Suppl):15.e11-15.e13, 2013

4. Ashour AM, Elbabaa SK, Caputy AJ, Gragnaniello C: Navigation-guided endoscopic intraventricular injectable tumor model: cadaveric tumor resection model for neurosurgical training. World Neurosurg 96:261-266, 2016

5. Baby B, Singh R, Suri A, Dhanakshirur RR, Chakraborty A, Kumar S, et al: A review of virtual reality simulators for neuroendoscopy. Neurosurg Rev [epub ahead of print], 2019

6. Bodani VP, Breimer GE, Haji FA, Looi T, Drake JM: Development and evaluation of a patient-specific surgical simulator for endoscopic colloid cyst resection. J Neurosurg [epub ahead of print June 28, 2019; DOI: 10.3171/2019.4.JNS183184]

7. Breimer GE, Bodani V, Looi T, Drake JM: Design and evaluation of a new synthetic brain simulator for endoscopic third ventriculostomy. J Neurosurg Pediatr 15:82-88, 2015

8. Breimer GE, Haji FA, Bodani V, Cunningham MS, LopezRios AL, Okrainec A, et al: Simulation-based education for endoscopic third ventriculostomy: a comparison between virtual and physical training models. Oper Neurosurg (Hagerstown) 13:89-95, 2017

9. Breimer GE, Haji FA, Cinalli G, Hoving EW, Drake JM: Validity evidence for the Neuro-Endoscopic Ventriculostomy Assessment Tool (NEVAT). Oper Neurosurg (Hagerstown) 13:60-68, 2017

10. Choudhury N, Gélinas-Phaneuf N, Delorme S, Del Maestro R: Fundamentals of neurosurgery: virtual reality tasks for training and evaluation of technical skills. World Neurosurg 80:e9-e19, 2013

11. Coelho G, Kondageski C, Vaz-Guimarães Filho F, Ramina R, Hunhevicz SC, Daga F, et al: Frameless image-guided neuroendoscopy training in real simulators. Minim Invasive Neurosurg 54:115-118, 2011

12. Coelho G, Zymberg S, Lyra M, Zanon N, Warf B: New anatomical simulator for pediatric neuroendoscopic practice. Childs Nerv Syst 31:213-219, 2015

13. Delorme S, Laroche D, DiRaddo R, Del Maestro RF: NeuroTouch: a physics-based virtual simulator for cranial microneurosurgery training. Neurosurgery 71 (1 Suppl Operative):32-42, 2012

14. Fernandez-Miranda JC, Barges-Coll J, Prevedello DM, Engh J, Snyderman C, Carrau R, et al: Animal model for endoscopic neurosurgical training: technical note. Minim Invasive Neurosurg 53:286-289, 2010

15. Filho FVG, Coelho G, Cavalheiro S, Lyra M, Zymberg ST: Quality assessment of a new surgical simulator for neuroendoscopic training. Neurosurg Focus 30(4):E17, 2011

16. Garling RJ, Jin X, Yang J, Khasawneh AH, Harris CA: Lowcost endoscopic third ventriculostomy simulator with mimetic endoscope. J Neurosurg Pediatr 22:137-146, 2018

17. Gragnaniello C, Gagliardi F, Chau AM, Nader R, Siu A, Litvack Z, et al: Intracranial injectable tumor model: technical advancements. J Neurol Surg B Skull Base 75:301-308, 2014

18. Haji FA, Dubrowski A, Drake J, de Ribaupierre S: Needs assessment for simulation training in neuroendoscopy: a Canadian national survey. J Neurosurg 118:250-257, 2013 
19. Jaimovich SG, Bailez M, Asprea M, Jaimovich R: Neurosurgical training with simulators: a novel neuroendoscopy model. Childs Nerv Syst 32:345-349, 2016

20. Manson A, Poyade M, Rea P: A recommended workflow methodology in the creation of an educational and training application incorporating a digital reconstruction of the cerebral ventricular system and cerebrospinal fluid circulation to aid anatomical understanding. BMC Med Imaging 15:44, 2015

21. McGaghie WC, Issenberg SB, Cohen ER, Barsuk JH, Wayne DB: Does simulation-based medical education with deliberate practice yield better results than traditional clinical education? A meta-analytic comparative review of the evidence. Acad Med 86:706-711, 2011

22. Ryan JR, Chen T, Nakaji P, Frakes DH, Gonzalez LF: Ventriculostomy simulation using patient-specific ventricular anatomy, 3D printing, and hydrogel casting. World Neurosurg 84:1333-1339, 2015

23. Schwab B, Hungness E, Barsness KA, McGaghie WC: The role of simulation in surgical education. J Laparoendosc Adv Surg Tech A 27:450-454, 2017

24. Thieringer FM: 3D Print Lab. Universitätsspital Basel (https://www.unispital-basel.ch/ueber-uns/bereiche/ medizinische-querschnittsfunktionen/kliniken-instituteabteilungen/departement-radiologie/kliniken-institute/klinikfuer-radiologie-und-nuklearmedizin/zuweiser/3d-print-lab/) [Accessed January 17, 2020]

25. Waran V, Narayanan V, Karuppiah R, Thambynayagam HC, Muthusamy KA, Rahman ZAA, et al: Neurosurgical endoscopic training via a realistic 3-dimensional model with pathology. Simul Healthc 10:43-48, 2015
26. Zymberg S, Vaz-Guimarães Filho F, Lyra M: Neuroendoscopic training: presentation of a new real simulator. Minim Invasive Neurosurg 53:44-46, 2010

\section{Disclosures}

Material and financial support was provided by Söring $\mathrm{GmbH}$ for the development of the tumor model and partial funding was provided for the authors' annual pediatric neurosurgery symposium, held at the University Children's Hospital in Basel, Switzerland, June 27-28, 2019.

\section{Author Contributions}

Conception and design: all authors. Acquisition of data: Licci. Analysis and interpretation of data: Licci, Soleman. Drafting the article: Licci, Thieringer. Critically revising the article: all authors. Reviewed submitted version of manuscript: Licci. Statistical analysis: Licci. Administrative/technical/material support: all authors. Study supervision: Guzman, Soleman.

\section{Supplemental Information \\ Videos \\ Video 1. https://vimeo.com/385232501.}

\section{Correspondence}

Maria Licci: University Hospital Basel, Switzerland. marialucia. licci@gmail.com. 